\title{
Synthesis and properties of poly(alkylene vanillate) polyesters, a new class of bio-based polymers
}

${ }^{1}$ Alexandra Zamboulis, ${ }^{2}$ Eleftheria Xanthopoulou, ${ }^{1}$ Lazaros Papadopoulos, ${ }^{1}$ Zoi Terzopoulou, ${ }^{1}$ Dimitrios N. Bikiaris, ${ }^{2}$ George Z. Papageorgiou

[1] Department of Chemistry, Aristotle University of Thessaloniki, GR-541 24, Thessaloniki, Greece

[2] Chemistry Department, University of Ioannina, P.O. Box 1186, GR-45110, Ioannina, Greece

Modern societies are striving to adopt a more environmental-friendly lifestyle, boosting the dynamics of biobased polymers. In this context, vanillic acid, a lignin-derived aromatic hydroxy-acid, has recently attracted interest in the preparation of polyesters. These polyesters would emulate poly(ethylene terephthalate) with the advantage of being produced by nonedible renewable biomass.
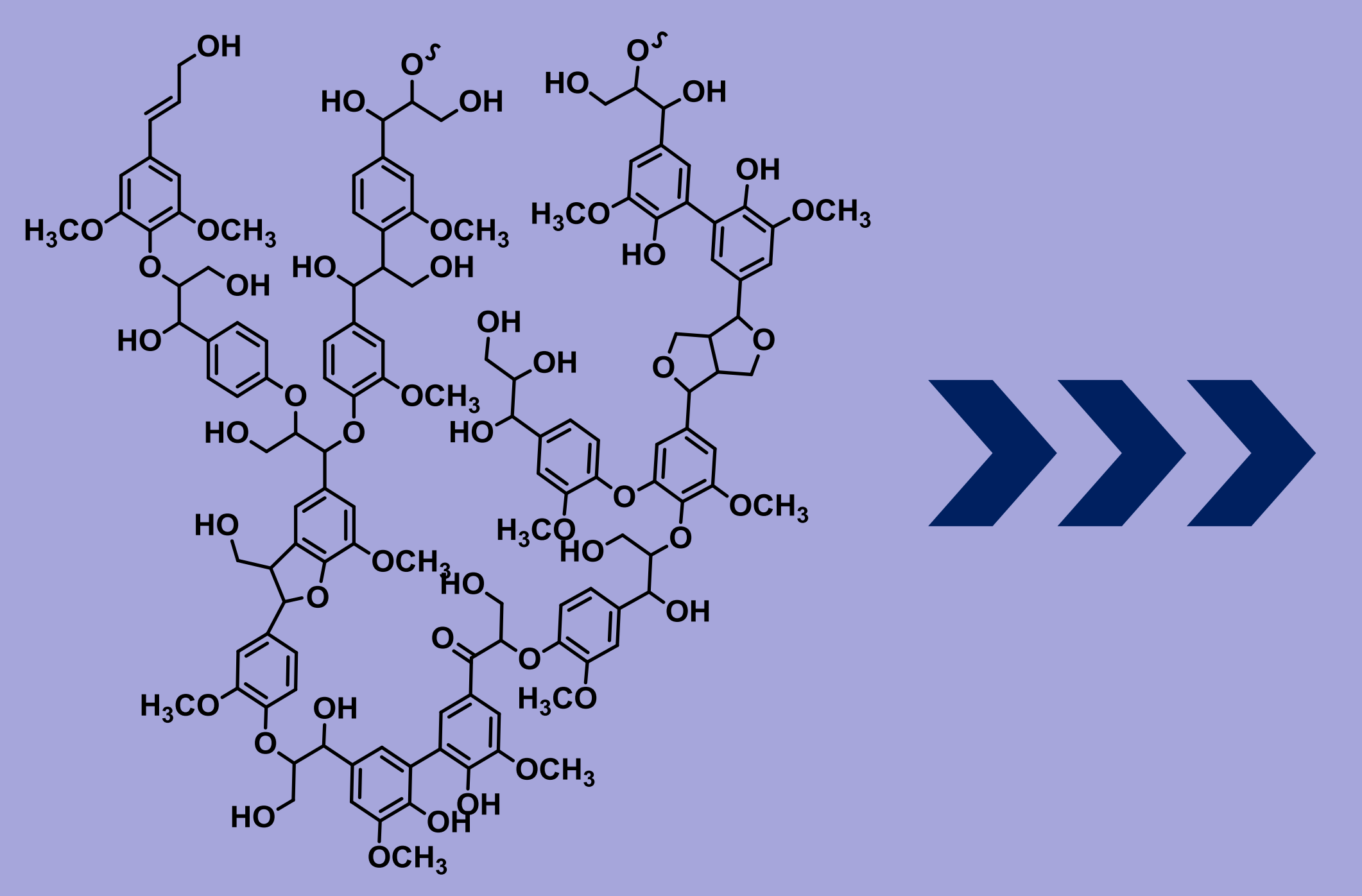

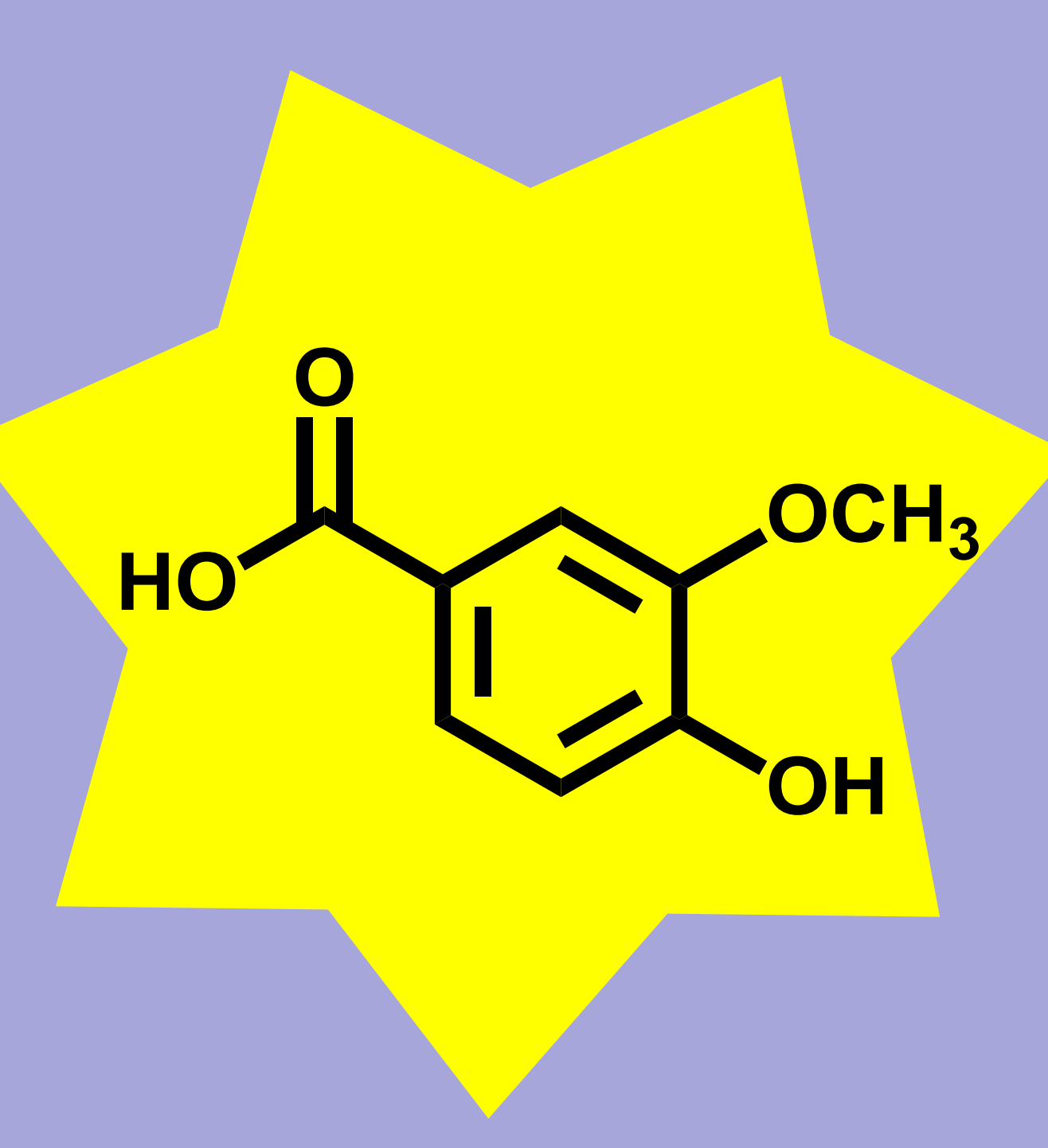

The phenolic hydroxyl group was etherified via a Williamson reaction, with two different $\omega$-chloroalcohols, to synthesize two $\omega$ hydroxy-alkylene vanillic acids. These hydroxy-acids were further polymerized by two-step melt polycondensation
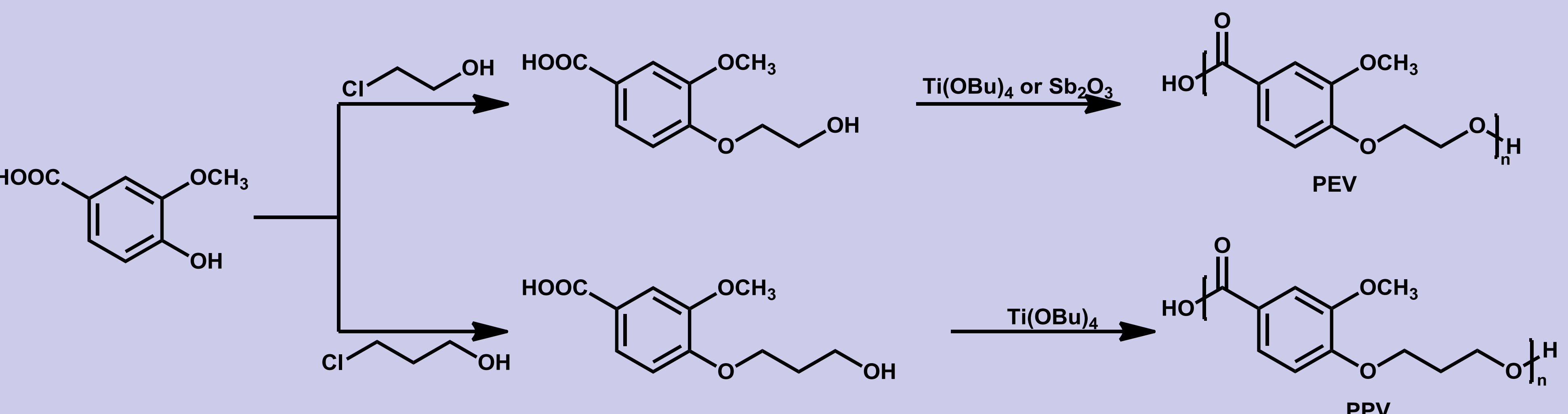

$$
\text { (1) }
$$

Novel poly(ethylene vanillate)-poly(ethylene furanoate) copolymers (PEFV) were synthesized by reacting 2-hydroxyethylene vanillic acid with PEF oligomers.

Structural characterization by infra-red spectroscopy and nuclear magnetic spectroscopy: successful synthesis of all polyesters.

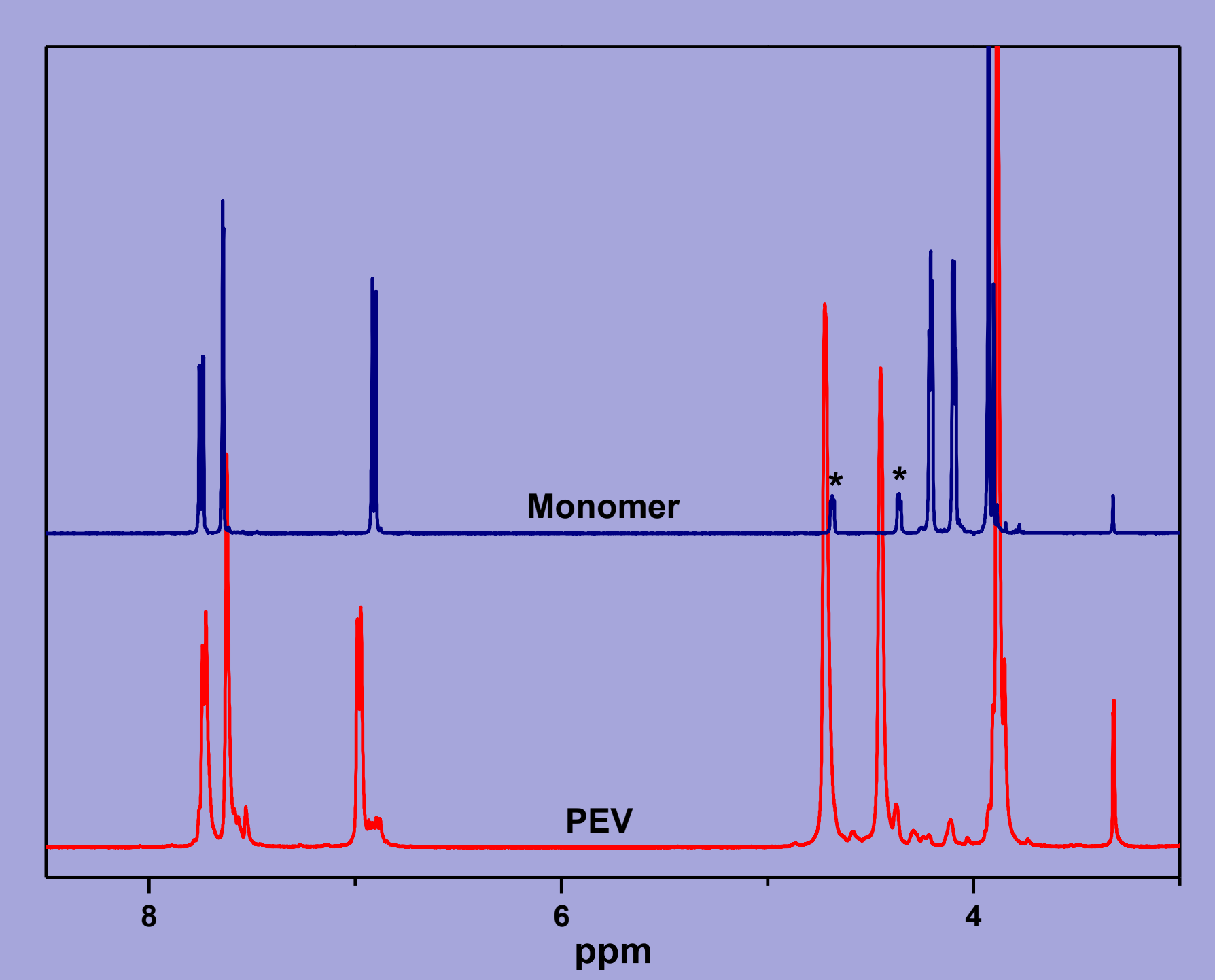

${ }^{1} \mathrm{H}$ NMR spectrum of PEV and its monomer

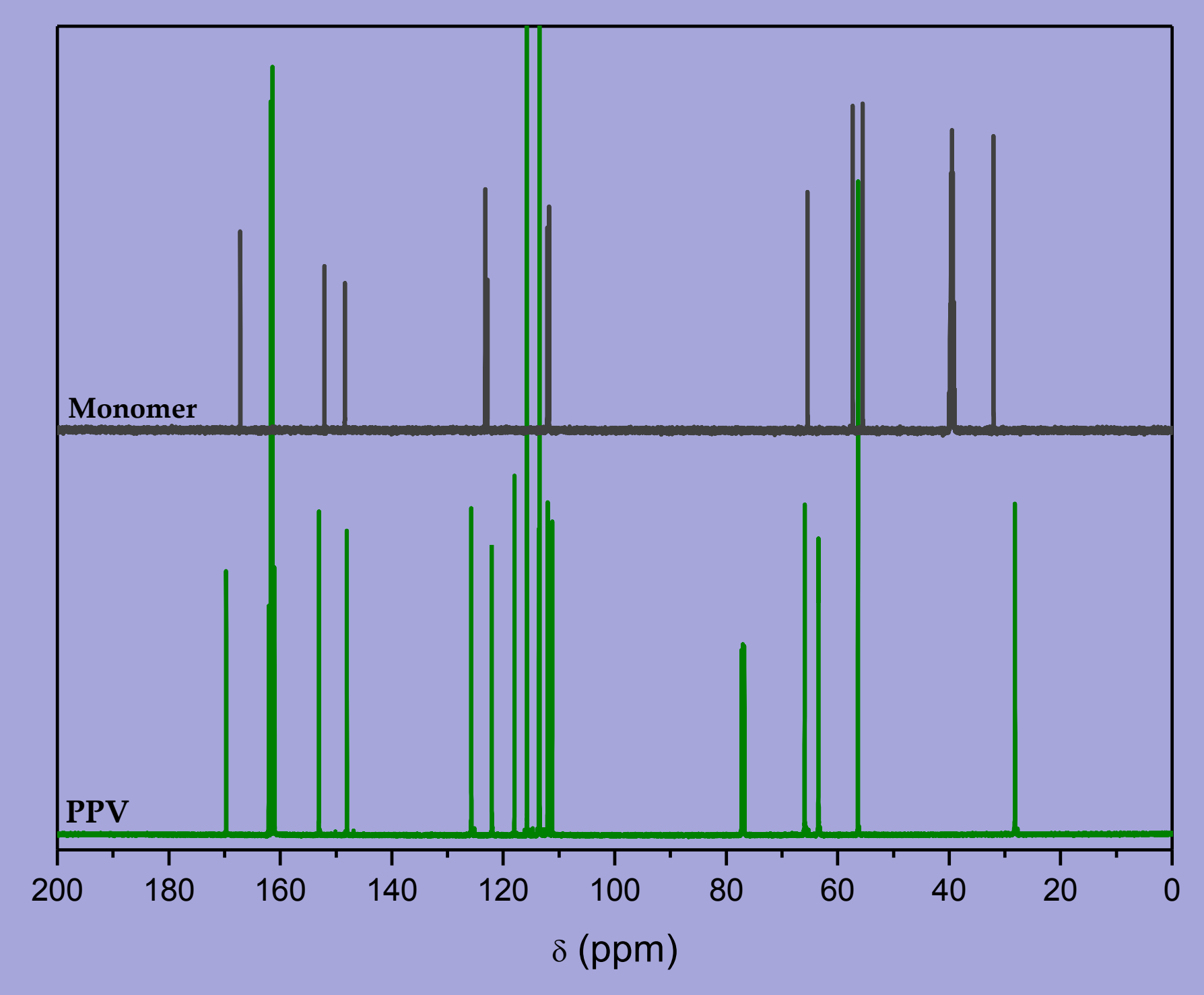

${ }^{13} \mathrm{C}$ NMR spectrum of PPV and its monomer

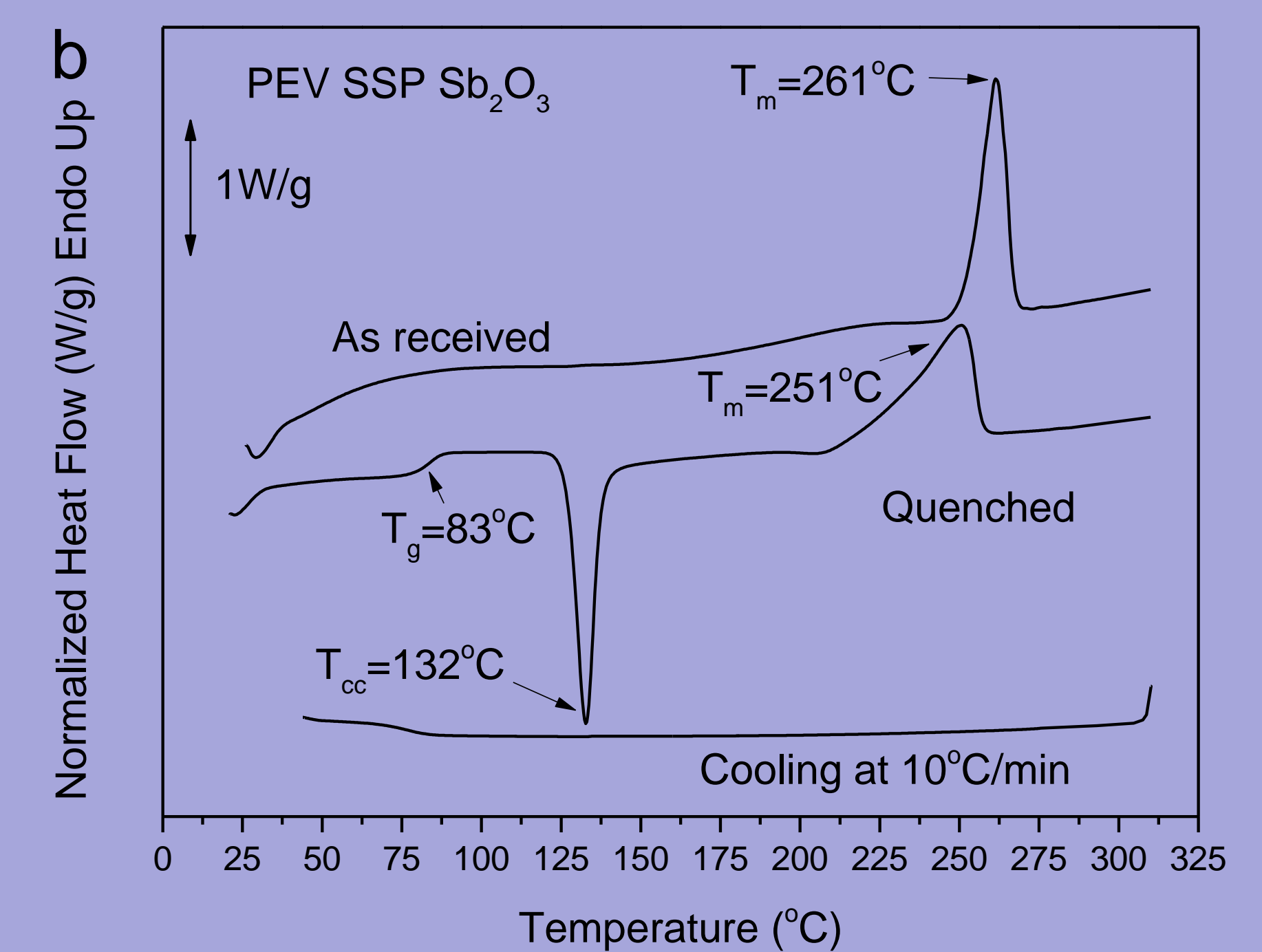

DSC scans of PEV

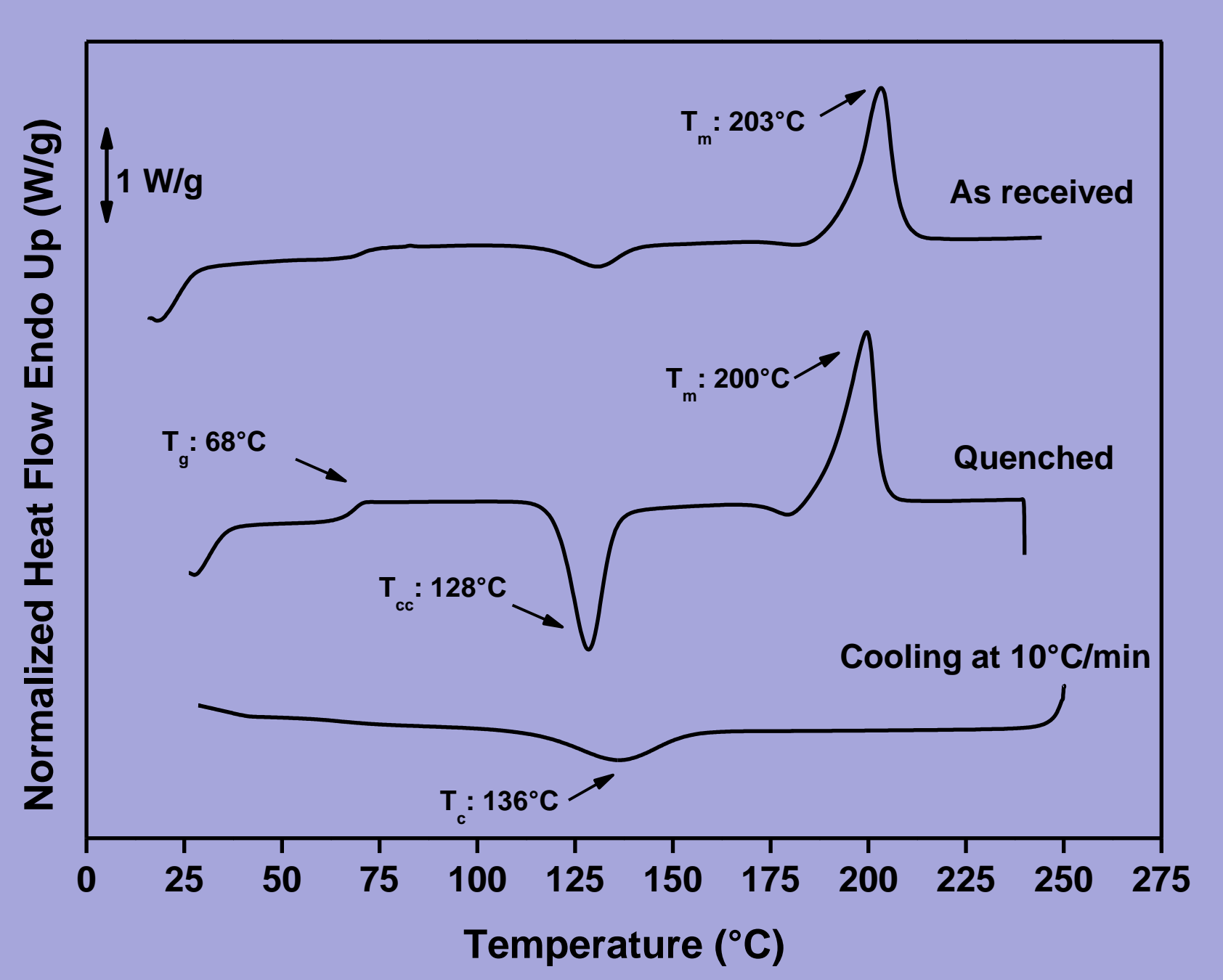

DSC scans of PPV

Equilibrium melting temperatures were estimated for both polymers, as well as the enthalpy and entropy of fusion of the $100 \%$ crystalline polymers.
The spherulitic morphology at different crystallization temperatures was studied by polarized light microcopy. A coarsening of spherulites is observed at higher temperatures.
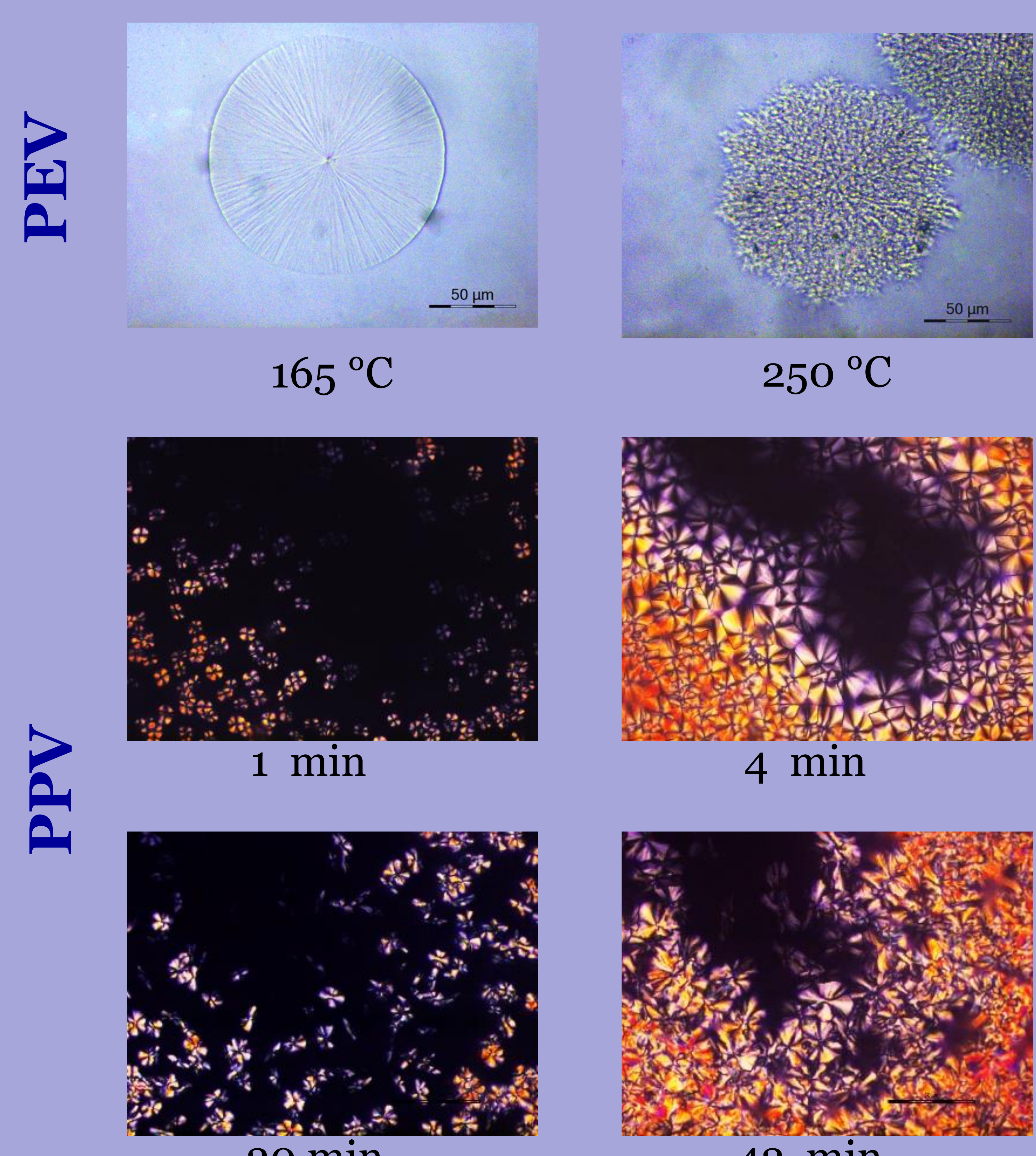

$20 \mathrm{~min}$
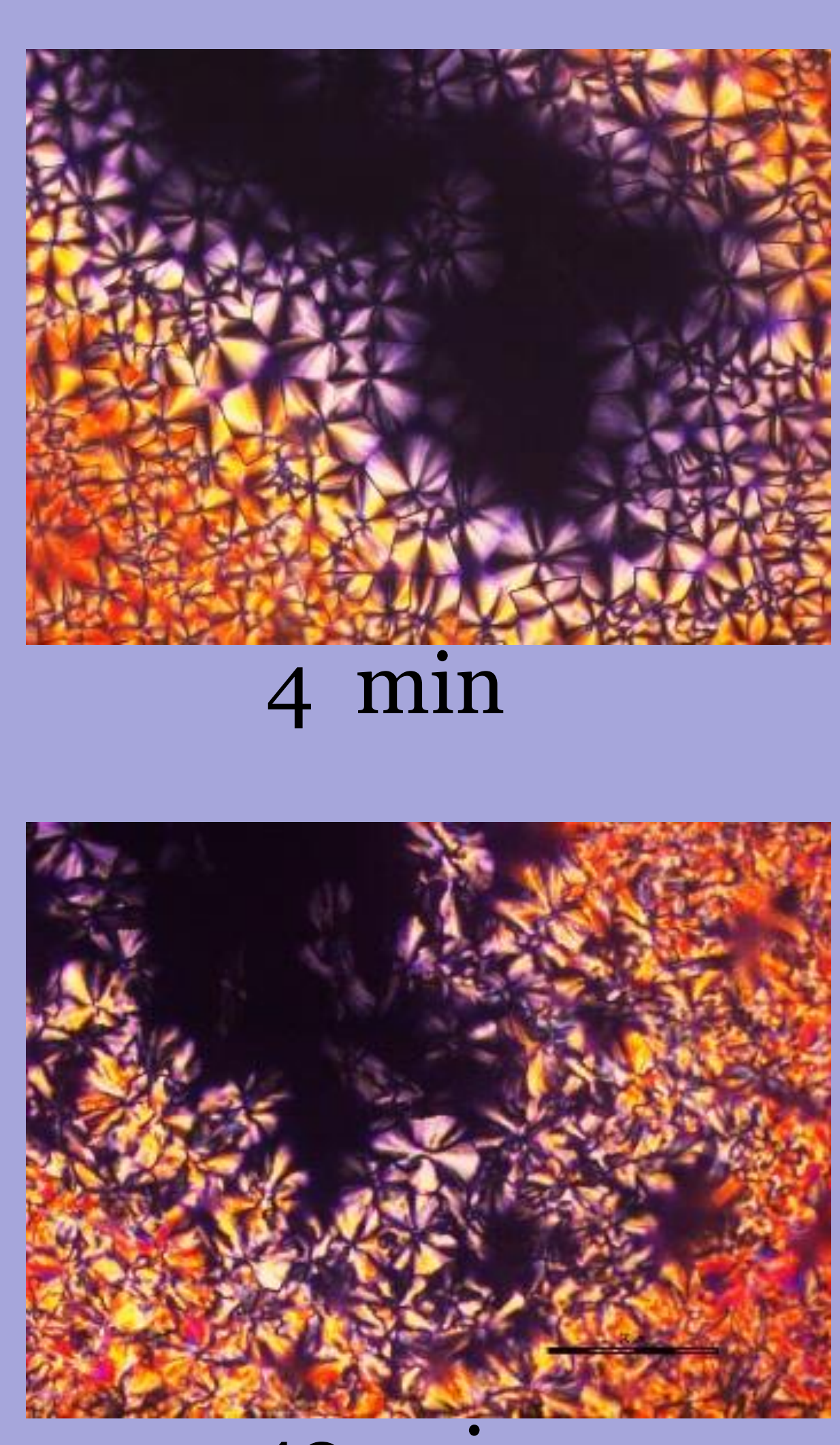

$190{ }^{\circ} \mathrm{C}$

- High molecular weight alipharomatic polyesters were synthesized from vanillic acid.

- Thermal properties and crystallization behavior was investigated.

- Mechanical properties were studied by nanoindentation.

$\left.\sum\right\rangle \quad$ Polyesters with promising properties

- Copolymers with PEF are under study.

@ azamboulis@gmail.com OF THESSALONIKI 